\title{
Nomogram based on albumin and neutrophil-to-lymphocyte ratio for predicting postoperative complications after pancreaticoduodenectomy
}

\author{
Haoquan Huang", Chengli Wang", Fengtao Ji, Zhixiao Han, Hui Xu, Minghui Cao \\ Department of Anesthesiology, Sun Yat-Sen Memorial Hospital, Sun Yat-Sen University, Guangzhou, China \\ Contributions: (I) Conception and design: H Huang, C Wang; (II) Administrative support: M Cao; (III) Provision of study materials or patients: H \\ Huang, F Ji, H Xu; (IV) Collection and assembly of data: H Huang, Z Han; (V) Data analysis and interpretation: H Huang, F Ji; (VI) Manuscript \\ writing: All authors; (VII) Final approval of manuscript: All authors. \\ "These authors contributed equally to this work. \\ Correspondence to: Minghui Cao. Department of Anesthesiology, Sun Yat-Sen Memorial Hospital, Sun Yat-Sen University, 107 Yanjiang West Road, \\ Guangzhou 510120, China. Email: caomh@mail.sysu.edu.cn.
}

Background: The aim of this study was to identify a preoperative inflammatory marker with the most
predictive value for postoperative complications after pancreaticoduodenectomy (PD). We then combined it
with other perioperative variables to construct and validate a nomogram for complications after PD. Methods: A total of 223 patients who received PD from January 2014 to July 2019 at a high-volume ( $>60 \mathrm{PDs} / \mathrm{year}$ ) pancreatic centers in China were included in this retrospective study. All of the PDs were performed by the same surgeon who is beyond the learning curve with more than 100 PDs over the previous 3 years before 2014. 15 preoperative inflammatory markers were collected, including neutrophils, lymphocytes, high-sensitivity C-reactive protein and lactic dehydrogenase. The inflammatory markers' predicting abilities for complications were analyzed by calculating the values of an area under the curve (AUC). The complications included surgical complications (such as pancreatic fistula, delayed gastric emptying and bile leakage) and medical complications (such as sepsis, pneumonia, urinary tract infection, acute heart failure and acute liver failure) in this study. Univariable and multivariable logistic regression analyses were performed to investigate the perioperative features for independent risk factors for complications after PD. Nomograms with or without the most predictive inflammatory for complications were subsequently developed based on multivariable logistic regression using Akaike information criterion. Nomograms' performance was quantified and compared in terms of calibration and discrimination. We studied the utility of the nomograms using decision curve analysis.

Results: The albumin/ NLR score (ANS) exhibited the highest AUC value (0.616) for predicting postoperative complications. ANS and approach method were identified as independent risk factors for complications. The nomogram with ANS had higher C-index (0.725) and better calibration. The NRI compared between nomograms was 0.160 (95\% CI: 0.023-0.296; $\mathrm{P}=0.022$ ). By decision curve analysis, the model with ANS had higher clinical value.

Conclusions: The ANS is a useful predictor and an independent risk factor for postoperative complications after PD. The nomogram with ANS was constructed with better performance and more clinical benefit for predicting postoperative complications.

Keywords: Nomogram; inflammatory maker; postoperative complications; pancreaticoduodenectomy (PD); albumin and neutrophil-to-lymphocyte ratio

Submitted Oct 28, 2020. Accepted for publication Jan 20, 2021.

doi: $10.21037 /$ gs-20-789

View this article at: http://dx.doi.org/10.21037/gs-20-789 


\section{Introduction}

Pancreaticoduodenectomy (PD) is a complex surgery, which is a common treatment method for both malignant tumors and benign diseases arising from the periampullary lesions. With improved surgical techniques and perioperative care, mortality rate after PD has been decreased to less than 5\% $(1,2)$. However, the postoperative morbidity rate of PD remains relatively high, ranging from $30-50 \%(3,4)$. The main complications following PD include pancreatic fistula (PF) (5), delayed gastric emptying (DGE) (6), pneumonia and systemic sepsis (4). The occurrence of complications is significantly associated with prolonged hospital length of stay and increased medical costs (7-9). Therefore, identifying risk factors for postoperative complications is of great importance in order to develop preventive and treatment strategies.

Previously, several risk factors for postoperative complications after PD have been identified, including age, sex, functional status and several abnormal laboratory results (4,5,10-12). Most of the relevant researches merely focused on PF $(5,11)$ and DGE $(6,12)$. However, since PD is an extensive abdominal surgical procedure, other adverse events, included pneumonia and sepsis, should also be taken into consideration. To identify risk factors for predicting a serious of complications after PD, one study has developed a risk calculator with good discriminatory performance (4). However, the predictive model was constructed with more than twenty variables (4). A more concise model is needed to predict complications after PD. In addition, since PD is one of the most complicate operations in general surgery, the prognosis may differ obviously for different surgeons because they are at different stages of the learning curve (13). For the related researches $(4,5,10,12)$, the cases were performed by different surgeons and thus, there was bias influencing the accuracy of the predicting model.

There is increasing evidence that systemic inflammatory response markers, included neutrophil-to-lymphocyte ratio (NLR), platelet-to-lymphocyte ratio (PLR) and prognostic nutritional index (PNI), are significantly associated with postoperative complications and prognosis in patients undergoing different surgeries (14-18). Albumin, a negative acute phase protein, decreases when inflammation or other situation occurs, included increased age and malnutrition (19-21). Preoperative malnutrition has been associated with several poor postoperative outcomes, including infection and mortality (4,21). A growing number of studies have shown that preoperative hypoalbuminemia has predictive value for postoperative complications in different surgical fields $(4,21)$. Therefore, if the systemic inflammatory marker is combined with albumin, the predictive value of it might increase. The Albumin/ NLR Score (ANS) combines albumin and NLR. Specially, albumin values below the optimal cutoff value, which is determined by the Youden index, are given 1 point. As for NLR, values over its optimal cutoff value are given 1 point. Each patient had an ANS score ranging from 0 to 2 . Recently, several studies demonstrated ANS played an important role in predicting complication and prognosis for patients with oral and gastric cancer (22-24). However, there is still no study assessing whether the simple and novel ANS has predictive ability for complications in patients with PD. Thus, in order to build a simple and reliable predictive model for postoperative complications after $\mathrm{PD}$, a preoperative inflammatory marker with the most predictive value should be found out and subsequently incorporated into the predictive model.

Nomogram is a statistical tool employed to predict an individual's particular outcome, which has been widely used in clinical practice $(22,24,25)$. The aim of this study was to find out the most predictive inflammatory marker and subsequently combine it with other perioperative variables to develop a nomogram to predict the likelihood of postoperative complications after PD, using data from one of the high-volume ( $>60 \mathrm{PDs} /$ year) pancreatic centers in China $(13,26)$.

We present the following article in accordance with the STROBE reporting checklist (available at http://dx.doi. org/10.21037/gs-20-789).

\section{Methods}

\section{Patients}

The study was conducted in accordance with the Declaration of Helsinki (as revised in 2013). The study was approved by institutional ethics committee of Sun YatSen Memorial Hospital (NO. SYSEC-KY-KS-2020-186) and individual consent for this retrospective analysis was waived. Patients who underwent classic Whipple PD for any indication by the same surgeon in our hospital between January 2014 and July 2019 were included. The surgeon had performed $>100$ PDs over the previous 3 years before 2014, and thus, considered to be beyond the learning curve $(13,26)$. The exclusion criteria included age below 18 years, emergency surgery and missing data. Emergency surgery was defined as an emergency procedure performed within 
$12 \mathrm{~h}$ after admission or after the onset of related symptoms. Missing data in this study was defined as any unknown variables for demographic characteristics, preoperative laboratory tests, surgical variables and postoperative variables.

\section{Data collection}

Patient demographic characteristics including age, sex, weight, American Society of Anesthesiologists (ASA) status and comorbidities (hypertension, diabetes, coronary heart disease, stroke, chronic obstructive pulmonary disease (COPD) and others) were obtained.

Preoperative laboratory tests including hemoglobin, platelet, neutrophils, lymphocytes, international normalized ratio (INR), high-sensitivity C-reactive protein (hsCRP), total bilirubin (T-Bil), alanine aminotransferase (ALT), lactic dehydrogenase (LDH), alkaline phosphatase (ALP), cholinesterase (CHE) and albumin were also collected. The neutrophil-to-lymphocyte ratio (NLR), platelet-tolymphocyte ratio (PLR) and ANS were calculated. Youden index integrates sensitivity and specificity information under circumstances that emphasize both sensitivity and specificity. The maximum value of the index provides the best tradeoff between sensitivity and specificity, and it can be used as a cut-off for numerical diagnostic tests $(22,27)$. Therefore, Youden index was employed in our study in order to find out the cut-off value that represents the maximum potential effectiveness of albumin and NLR. The cut-off value for albumin was $37.45 \mathrm{~g} / \mathrm{L}$ and the NLR's cur-off value was 3.7758 in this study. ANS is a combination of albumin and NLR, there are three potential types of combination for each patient's ANS, including low albumin plus low NLR, low albumin plus high NLR and high albumin plus high NLR. Both low albumin level and high NLR level have been reported to be associated with higher rate of complications after surgeries (16-18,21). In order to assign scores to three combination of albumin and NLR, we set the rule that the higher ANS value, the higher complication rate. This rule is consistent with previous related studies (22-24). Therefore, values of albumin below $37.45 \mathrm{~g} / \mathrm{L}$ were given 1 point. As for NLR, values over 3.7758 were given 1 point. Each patient had an ANS score ranging from 0 to 2 .

Surgical variables including approach method, estimated blood loss, intraoperative transfusion, total fluid, total crystalloid, total colloid, intraoperative infusion rate, intraoperative urine and operation time were recorded.
Postoperative variables included intensive care unit (ICU) admission, Clavien-Dindo severity classification of complications. The postoperative complications included PF, DGE, bile leakage, postoperative bleeding, gastrointestinal anastomotic obstruction, wound infection, wound dehiscence, abdominal infection, sepsis, pneumonia, urinary tract infection, acute heart failure and acute liver failure. Occurrence of pancreatic fistula was classified according to the ISGPF classification as Biochemical Fistula, B, or C (27); Delayed gastric emptying was classified based on the International Study Group on Pancreatic Surgery guidelines as A, B, or C (28); Postoperative bleeding was classified according to the International Study Group on Pancreatic Surgery guidelines (29). Each postoperative complication was classified according to Clavien-Dindo severity classification (30). The primary outcome of this study was the occurrence of postoperative complication, which was defined as a Clavien-Dindo classification grade II or higher complication.

\section{Comparison of AUC values among laboratory tests}

To compare the discriminative abilities for postoperative complications of the laboratory tests, a receiver operating characteristic (ROC) curve analysis was employed to calculate the area under the curve (AUC) of each parameter. After comparison, ANS was selected for further analyses because it had the highest AUC value. Subsequently, in order to evaluate whether ANS has significant impact on postoperative complications, comparisons of the rate and the severity of complication among three subgroups according to the ANS stages was carried out. Continuous variables were assessed by one-way Analysis of Variance (ANOVA) or Kruskal-Wallis test according to the normality and homogeneity for variables. Categorical variables were analyzed by chi-square test or Fisher exact test according to the frequencies of variables.

\section{Univariable and multivariable logistical regressions}

Univariable and multivariable logistical regressions were employed to identify risk factors for postoperative complications. Variance inflation factor (VIF) was used to detect multicollinearity in regression analysis, with a reference value of 10. The values of VIF of neutrophils, lymphocytes, platelet, albumin, PLR, NLR, ANS, total fluid, total crystalloid, total colloid and intraoperative urine and intraoperative infusion rate were higher than 10 , 
indicating these variables were correlated. In order to retain the most important variable among the collinear variables, we refer to the results of univariable logistic regression. Therefore, neutrophils, lymphocytes, albumin, PLR, NLR, total fluid, total crystalloid, total colloid and intraoperative urine were excluded for multivariable logistical regression.

\section{Development and validation of the nomograms}

To build the predictive models, the Akaike information criterion (AIC) was employed with and without ANS to select variables through a backward step-down process from the full multivariable regression model. Selected variables were incorporated into the nomograms to predict the probability of postoperative complications for PD. The concordance index (C-index) was used to assess the discrimination of nomograms. Calibration curves were employed to determine the calibration. Each calibration curve was corroborated with 1,000 resamples conducted for validation, reducing the overfit bias which would overstate the accuracy of the nomogram. The C-indexes, calibration curves, net reclassification improvement (NRI) of two models were compared to assess the accuracy difference of two models. The decision curve analysis (DCA) was carried out to evaluate and compare the potential net benefit between the two predictive models (31).

\section{Statistical analyses}

Continuous variables were reported as median and interquartile range (IQR), which were assessed by $t$-test or Mann-Whitney $U$-test according to the distribution of variables. Categorical variables were reported as whole numbers and percentages, which were analyzed by chi-square test or Fisher exact test according to the frequencies of variables. The difference among patient demographic characteristics, preoperative laboratory tests, surgical variables and postoperative variables were compared between patients with and without postoperative complications.

All statistical analyses were performed through $\mathrm{R}$ software version 3.4.2 (Institute for Statistics and Mathematics, Vienna, Austria; https://www.r-project. org/). The development and validation of nomograms were performed with "rms" R package, and DCA was carried out using "rmda" $\mathrm{R}$ package. A two-tailed $\mathrm{P}$ value $<0.05$ was considered to be statistically significant.

\section{Results}

\section{Patients characteristics}

249 patients who underwent PD between January 2014 and July 2019 were extracted from the database. 26 patients were excluded because of missing information including preoperative laboratory tests (hemoglobin: $\mathrm{n}=3$; hsCRP: $n=22$ ) and surgical variables (operation time: $n=1$ ). After selection, 223 patients were included for analysis. $107(48.0 \%)$ patients developed at least one grade II or higher postoperative complication, which was defined as postoperative complication for subsequent analyses in this study. The grade II or higher complications included PF (38, 17.0\%), DGE (21,9.4\%), bile leakage (14, 6.3\%), postoperative bleeding $(20,9.0 \%)$, gastrointestinal anastomotic obstruction $(2,0.9 \%)$, wound infection (1, $0.4 \%)$, wound dehiscence $(1,0.4 \%)$, abdominal infection $(36,16.1 \%)$, sepsis $(7,3.1 \%)$, pneumonia $(29,13.0 \%)$, urinary tract infection $(2,0.9 \%)$, acute heart failure $(2,0.9 \%)$ and acute liver failure (2, $0.9 \%)$. Reoperation was carried out in $10(4.5 \%)$ patients. With regard to the ClavienDindo severity classification, while $84(37.7 \%)$ patients had grade II postoperative complications, 23 (9.9\%) patients had grade III or greater one.

Table 1 shows patients' demographic characteristics, preoperative laboratory tests, surgical variables and postoperative variables stratified by postoperative complications. Patients who developed complications were more likely to have higher neutrophils level [4.19 $(3.42-5.50) \times 10^{9} / \mathrm{L}$ vs. $\left.3.62(2.78-4.93) \times 10^{9} / \mathrm{L} ; \mathrm{P}=0.005\right]$, higher T-Bil level [48.70 (14.30-148.90) $\mu \mathrm{mol} / \mathrm{L} v s .21 .70$ (10.35-118.40) $\mu \mathrm{mol} / \mathrm{L} ; \mathrm{P}=0.017]$, higher LDH level [196.00 (168.00-237.00) U/L vs. 184.50 (158.00-225.25) U/L; $\mathrm{P}=0.042]$, lower albumin level [36.60 (31.70-40.50) $\mathrm{g} / \mathrm{L} v s$. 38.00 (33.73-41.48) g/L; $\mathrm{P}=0.101]$, higher NLR level [3.38 (2.15-4.64) vs. 2.44 (1.75-3.75); $\mathrm{P}=0.006]$ and higher ANS stage (ANS $=2: 29.0 \%$ vs. $17.2 \% ; \mathrm{P}=0.004$ ). In addition, patients in the postoperative complication-positive group were more prone to have open operation $(41.1 \%$ vs. $18.1 \%$; $\mathrm{P}<0.001)$, have larger amount of estimated blood loss $[150.00$ (100.00-400.00) vs. $100.00(50.00-200.00) \mathrm{mL} ; \mathrm{P}=0.006]$ and lager amount of intraoperative urine [400.00 (300.00-650.00) vs. 350.00 (200.00-495.00) mL; $\mathrm{P}=0.009]$.

In the present study, the rate of total laparoscopy, open and conversion to laparotomy was $62.3 \%, 29.1 \%$ and $8.5 \%$, respectively. In addition, there was significant difference for the complication rate of total laparoscopy, 
Table 1 Patients characteristics stratified by postoperative complications

\begin{tabular}{|c|c|c|c|c|}
\hline & $\begin{array}{l}\text { Complication-negative group } \\
\qquad(\mathrm{n}=116)\end{array}$ & $\begin{array}{l}\text { Complication-positive group } \\
\qquad(\mathrm{n}=107)\end{array}$ & $\begin{array}{l}\text { Statistic } \\
\text { value }\end{array}$ & $\mathrm{P}$ \\
\hline Age (years) & $60.00[50.25,64.88]$ & $62.00[54.00,68.00]$ & $-1.627^{a}$ & $0.104^{*}$ \\
\hline \multicolumn{5}{|l|}{ Sex } \\
\hline Female & $50(41.4 \%)$ & $41(40.2 \%)$ & $0.033^{c}$ & 0.856 \\
\hline \multicolumn{5}{|l|}{ ASA status } \\
\hline$|-I|$ & $24(20.7 \%)$ & $23(21.5 \%)$ & $0.022^{c}$ & 0.883 \\
\hline III-IV & 92 (79.3\%) & $84(78.5 \%)$ & & \\
\hline \multicolumn{5}{|l|}{ Preoperative } \\
\hline Hemoglobin (g/L) & $124.00[111.25,135.00]$ & $120.00[104.00,136.00]$ & $1.548^{\mathrm{b}}$ & 0.123 \\
\hline Platelet (×10\%/L) & $254.00[199.25,295.00]$ & $256.00[205.00,342.00]$ & $-1.194^{\mathrm{a}}$ & $0.233^{*}$ \\
\hline Neutrophils $\left(\times 10^{9} / L\right)$ & $3.62[2.78,4.93]$ & $4.19[3.42,5.50]$ & $-2.827^{\mathrm{a}}$ & $0.005^{\star}$ \\
\hline Lymphocytes (×10\%/L) & $1.52[1.17,1.89]$ & $1.54[1.16,1.86]$ & $-0.092^{a}$ & $0.926^{\star}$ \\
\hline INR & $1.04[0.98,1.10]$ & $1.03[0.97,1.10]$ & $-0.879^{a}$ & $0.380^{*}$ \\
\hline hsCRP (mg/L) & $3.87[1.22,14.37]$ & $5.66[2.19,17.51]$ & $-1.482^{\mathrm{a}}$ & $0.138^{*}$ \\
\hline Albumin (g/L) & $38.00[33.73,41.48]$ & $36.60[31.70,40.50]$ & $-1.639^{\mathrm{a}}$ & $0.101^{*}$ \\
\hline PLR & $162.73[116.87,239.22]$ & $173.17[129.49,262.05]$ & $-0.803^{\mathrm{a}}$ & $0.422^{*}$ \\
\hline NLR & $2.44[1.75,3.75]$ & $3.38[2.15,4.64]$ & $-2.742^{\mathrm{a}}$ & $0.006^{*}$ \\
\hline \multicolumn{5}{|l|}{ ANS } \\
\hline 0 & $58(50.0 \%)$ & $31(29.0 \%)$ & $10.808^{c}$ & 0.004 \\
\hline 1 & 38 (32.8\%) & $45(42.1 \%)$ & & \\
\hline 2 & $20(17.2 \%)$ & $31(29.0 \%)$ & & \\
\hline \multicolumn{5}{|l|}{ Approach method } \\
\hline Total laparoscopy & $84(72.4 \%)$ & $55(51.4 \%)$ & $14.323^{c}$ & $<0.001$ \\
\hline Open & $21(18.1 \%)$ & $44(41.1 \%)$ & & \\
\hline Conversion to laparotomy & $11(9.5 \%)$ & $8(7.5 \%)$ & & \\
\hline
\end{tabular}

Table 1 (continued) 
Table 1 (continued)

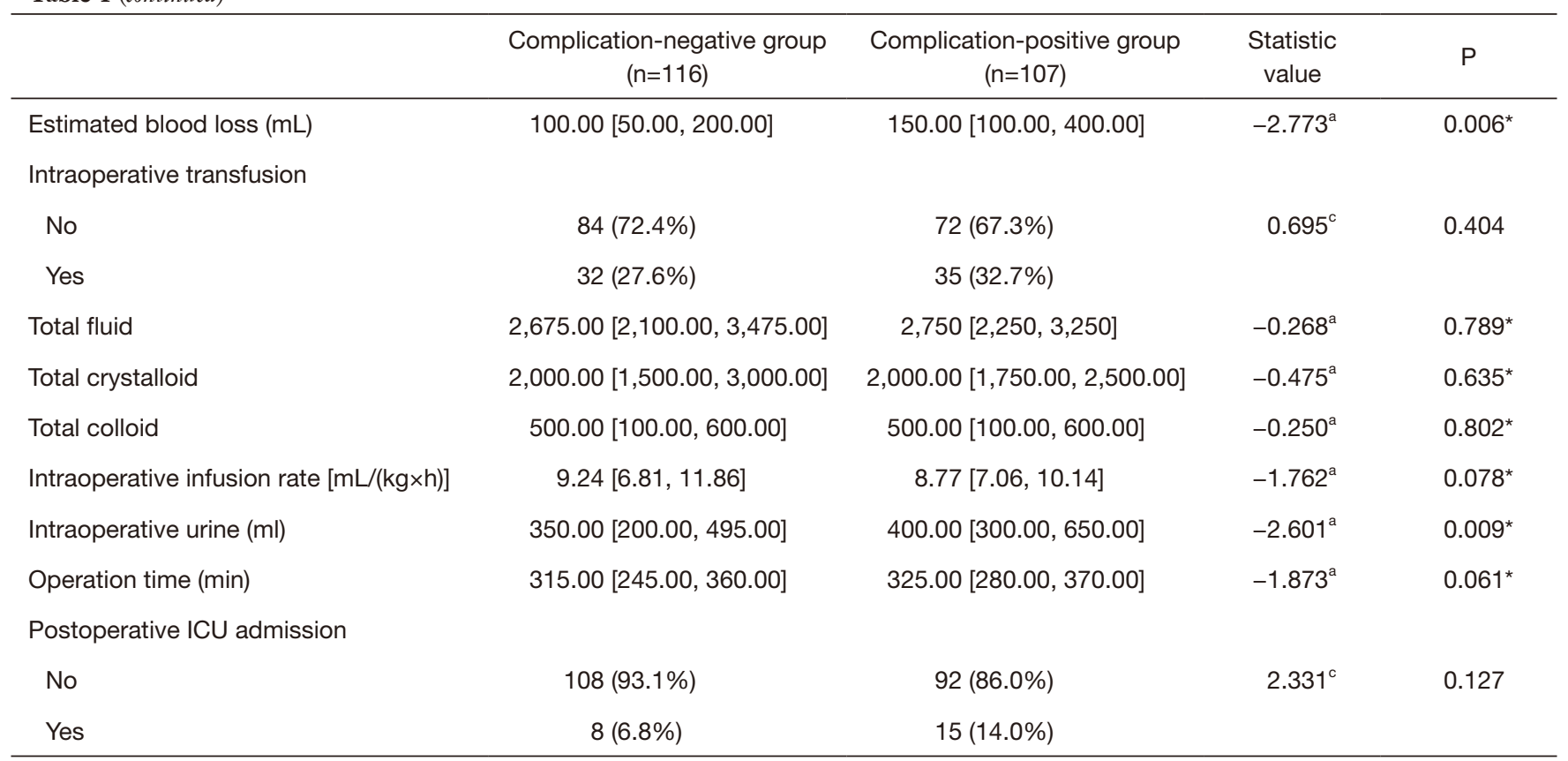

*, Mann-Whitney U test; ${ }^{a}, \mathrm{Z}$ value for Mann-Whitney $\mathrm{U}$ test; ${ }^{\mathrm{b}}$, T value for $t$-test; ${ }^{\mathrm{c}}$, Chi-square value for chi-square test. Complication was defined as a Clavien-Dindo classification grade II or higher complication. ASA status, the American Society of Anesthesiologists physical status; INR, international normalized ratio; hsCRP, high-sensitivity C-reactive protein; T-Bil, total bilirubin; ALT, alanine aminotransferase; LDH, lactic dehydrogenase; ALP, alkaline phosphatase; CHE, cholinesterase; PLR, Platelet-to-lymphocyte ratio; NLR, Platelet-tolymphocyte ratio; ANS, Albumin/NLR Score; ICU, intensive care unit.

open and conversion to laparotomy with $39.6 \%, 67.7 \%$ and $42.1 \%$, respectively $(\mathrm{P}<0.001)$. Compared to patients with laparotomy, those who underwent total laparoscopy had significant lower rates of $\mathrm{PF}(12.9 \%$ vs. $27.7 \%, \mathrm{P}=0.017)$ and DGE (5.8\% vs. $15.4 \%, \mathrm{P}=0.046)$. The detailed description of complications stratified by approach method was shown in Table S1.

\section{Comparison of the predictive value of potential indicators}

Calculating the AUC value of each candidate parameter, the predictive ability among them were compared. As shown in Table 2, the AUC values of ANS, neutrophils, lymphocytes, platelet, NLR and PLR were 0.616, 0.598, $0.504,0.546,0.567$ and 0.531 , respectively. Since ANS was the most predictive one with the AUC value 0.616 among 15 candidate parameters, it was selected to further analysis. Table 3 shows both the rate of postoperative complication and the Clavien-Dindo severity classification of complication were significantly different among different ANS stages.

\section{Univariable and multivariable analyses between the complication-positive and complication-negative groups}

Table 4 shows the univariable and multivariable analyses between patients with and without postoperative complications. In univariable analysis, neutrophils, T-Bil, LDH, ALP, ANS, approach method, intraoperative infusion rate, intraoperative urine and operation time were significantly associated with postoperative complications after PD. In the full multivariable analysis, ANS and approach method were identified as significantly independent risk factors for postoperative complications after PD.

\section{Construction of nomograms}

When ANS was not included in the AIC-based multivariable analysis, age, preoperative $\mathrm{LDH}$, approach method, estimated blood loss and intraoperative infusion rate were identified as independent risk factors for postoperative complications after PD (Table 5). When ANS was included in the analysis, preoperative LDH, ANS, approach method, 
intraoperative infusion rate and operation time were identified as independent risk factors for postoperative complications (Table 5). These identified risk factors were employed to construct Model 1 (nomogram without ANS)

Table 2 Comparison of the predictive value of potential indicators

\begin{tabular}{ll}
\hline & AUC \\
\hline ANS & 0.616 \\
Neutrophils & 0.598 \\
T-Bil & 0.592 \\
LDH & 0.579 \\
NLR & 0.567 \\
Albumin & 0.564 \\
ALP & 0.563 \\
hsCRP & 0.557 \\
CHE & 0.550 \\
Hemoglobin & 0.546 \\
PLR & 0.531 \\
INR & 0.534 \\
ALT & 0.519 \\
Platelet & 0.546 \\
Lymphocytes & 0.504 \\
\hline AUC, area under the curve; INR, &
\end{tabular}

AUC, area under the curve; INR, international normalized ratio; hsCRP, high-sensitivity C-reactive protein; T-Bil, total bilirubin; ALT, alanine aminotransferase; LDH, lactic dehydrogenase; ALP, alkaline phosphatase; CHE, cholinesterase; PLR, Plateletto-lymphocyte ratio; NLR, Platelet-to-lymphocyte ratio; ANS, Albumin/NLR Score. and Model 2 (nomogram with ANS), respectively (Figure 1).

\section{Comparison of predictive accuracy between two nomograms}

The C-indexes of the nomograms were 0.710 and 0.725 in Model 1 (nomogram without ANS) and Model 2 (nomogram with ANS), respectively. These results indicated that the discrimination ability of Model 2 was better than the ability of Model 1 . To assess the calibration, calibration curves were employed (Figure $2 A, B$ ). The calibration curves demonstrated that compared with Model 1 (Figure 2A), there was higher agreement between the nomogram prediction and actual outcomes in Model 2 (Figure 2B). In addition, the NRI compared between the two models was 0.160 (95\% CI: 0.023-0.296) and there was a significant difference $(\mathrm{P}=0.022)$, indicating that Model 2 has better predictive accuracy than Model 1.

\section{Evaluation of clinical benefit between two nomograms}

In order to evaluate the improvement of the nomograms bringing on decision-making, DCA for each model was built (Figure 2C). It was assumed that the treatment decision for a patient was made if the predictive probability was above the threshold probability, while the predictive probability under the threshold would produce a decision otherwise. When the threshold probability was given between around $30 \%$ and $80 \%$, both of the models were applicable since the benefit of them were beyond the "treat all patients" strategy and "treat none" strategy. Compared with Model 1 (nomogram without ANS), there was a higher net benefit of Model 2 (nomogram with ANS) (Figure 2C).

Table 3 Patients characteristics stratified by ANS stages

\begin{tabular}{|c|c|c|c|c|c|}
\hline & ANS $=0(n=89)$ & ANS $=1(n=83)$ & ANS $=2(n=51)$ & Statistic value & $\mathrm{P}$ \\
\hline Complication & & & & $13.011^{\mathrm{a}}$ & $0.001^{*}$ \\
\hline No & $59(66.3 \%)$ & $38(45.8 \%)$ & $19(37.3 \%)$ & & \\
\hline Yes & $30(33.7 \%)$ & $45(54.2 \%)$ & $32(62.7 \%)$ & & \\
\hline Clavien-Dindo severity classification of complication & & & & $13.411^{\mathrm{a}}$ & 0.009 \\
\hline Grade II & $24(27.0 \%)$ & $36(43.4 \%)$ & $24(47.1 \%)$ & & \\
\hline Grade III or greater & $6(6.7 \%)$ & $9(10.8 \%)$ & $8(15.7 \%)$ & & \\
\hline
\end{tabular}

Complication was defined as a Clavien-Dindo classification grade II or higher complication. ${ }^{\#}$ Chi-square test. ${ }^{a}$ Chi-square value for chisquare test. ANS, albumin/NLR score. 
Table 4 Univariable and multivariable logistic regression analysis of factors associated with complications

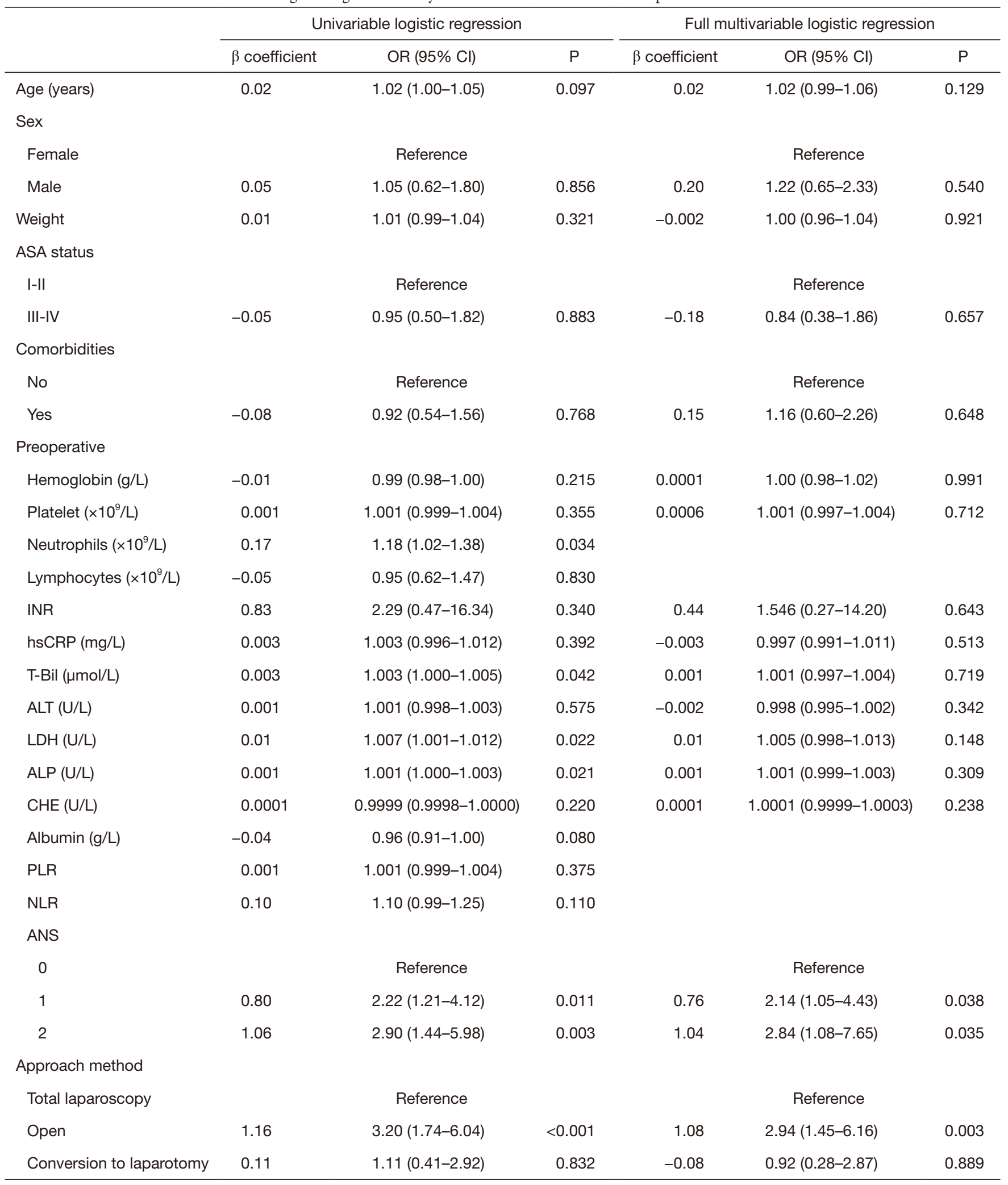

Table 4 (continued) 
Table 4 (continued)

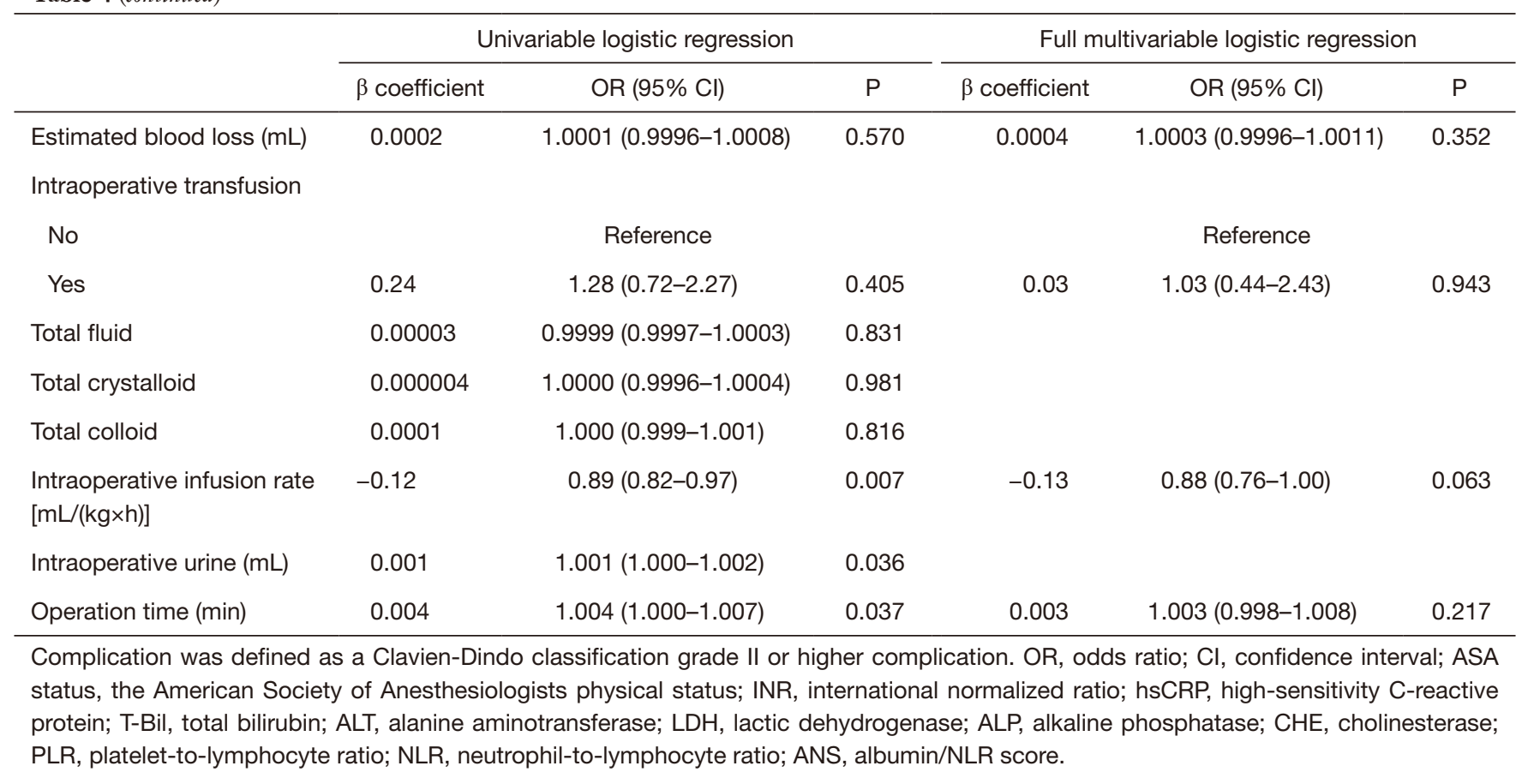

Table 5 Risk factors for postoperative complication derived from AIC-based multivariable logistic regression

\begin{tabular}{|c|c|c|c|c|c|c|}
\hline & \multicolumn{3}{|c|}{ Model 1} & \multicolumn{3}{|c|}{ Model 2} \\
\hline Age (years) & 0.02 & $1.02(1.00-1.05)$ & 0.089 & & NA & NA \\
\hline Preoperative LDH (U/L) & 0.01 & $1.007(1.001-1.013)$ & 0.025 & 0.01 & $1.006(1.000-1.012)$ & 0.061 \\
\hline \multicolumn{7}{|l|}{ ANS } \\
\hline 1 & & NA & NA & 0.70 & $2.02(1.04-3.95)$ & 0.038 \\
\hline 2 & & NA & NA & 0.99 & $2.69(1.27-5.84)$ & 0.011 \\
\hline \multicolumn{7}{|l|}{ Approach method } \\
\hline Total laparoscopy & & Reference & & & Reference & \\
\hline Estimated blood loss (mL) & 0.001 & $1.0005(0.9999-1.0011)$ & 0.109 & & NA & NA \\
\hline $\begin{array}{l}\text { Intraoperative infusion rate } \\
{[\mathrm{mL} /(\mathrm{kg} \times \mathrm{h})]}\end{array}$ & -0.14 & $0.87(0.79-0.96)$ & 0.005 & -0.09 & $0.91(0.83-1.00)$ & 0.045 \\
\hline Operation time (min) & & NA & NA & 0.003 & $1.003(0.999-1.007)$ & 0.111 \\
\hline
\end{tabular}

OR, odds ratio; $\mathrm{Cl}$, confidence interval; AIC, Akaike information criterion; LDH, lactic dehydrogenase; ANS, albumin/NLR score. 


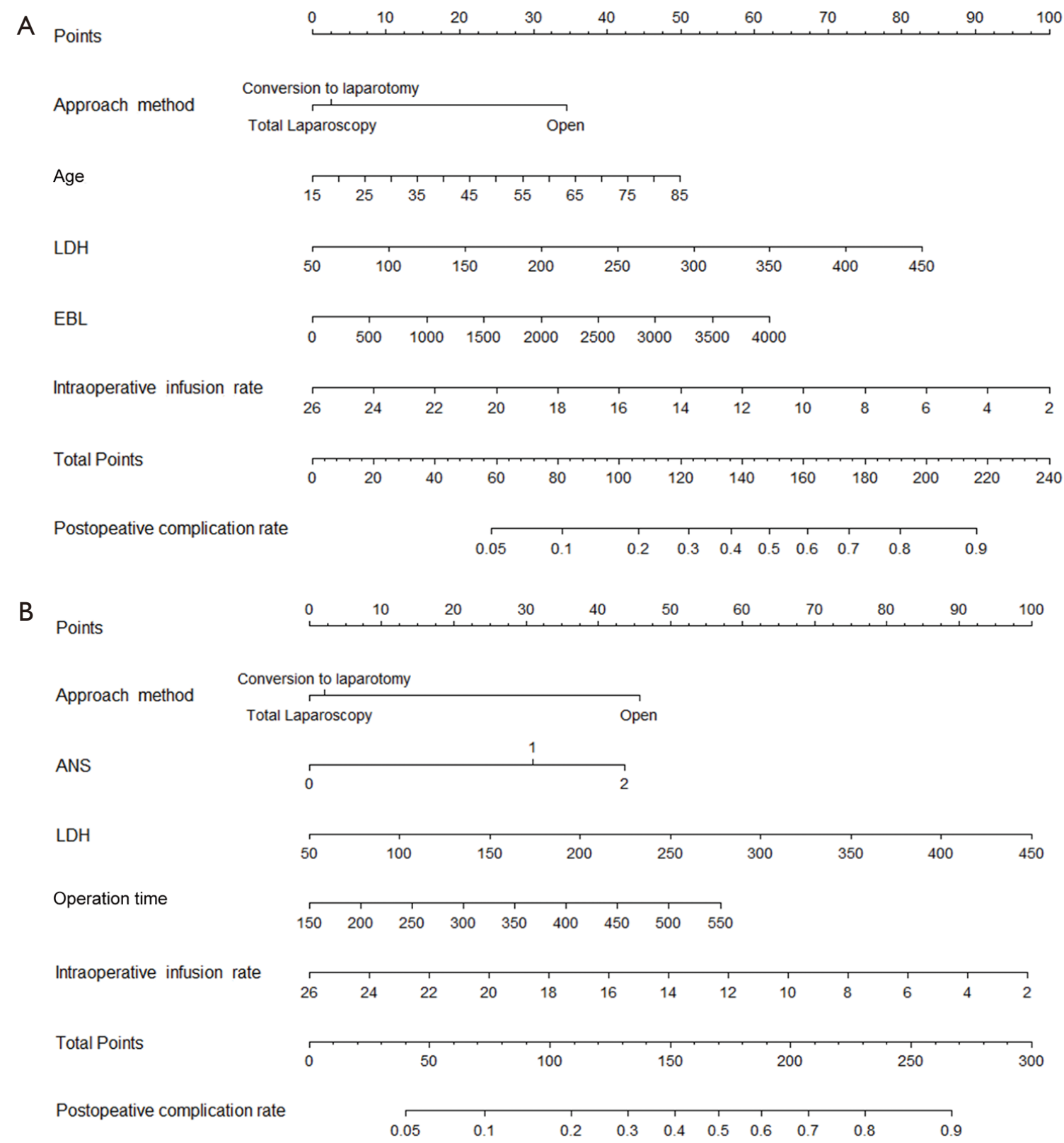

Figure 1 Nomograms derived from the AIC-based multivariate analyses. (A) The nomogram without ANS; (B) The nomogram incorporating ANS. AIC, Akaike information criterion; LDH, lactic dehydrogenase; EBL, estimated blood loss; ANS, albumin/NLR score.

\section{Discussion}

This study demonstrated that ANS had the highest predictive value for postoperative complications after PD among the 15 parameters. In addition, ANS was found to be an independent risk factor for postoperative complications in a multivariable logistic regression analysis. Combining ANS with other perioperative variables, this study further constructed a nomogram to predict postoperative complications, which showing good discrimination and good calibration. This applicable nomogram could be used to identify individuals at risk of postoperative complications, facilitating physicians' decision-making.

Previous studies showed that a couple of systemic inflammatory markers could be employed as predictive factor for complications after different surgeries $(14,17,18)$. 


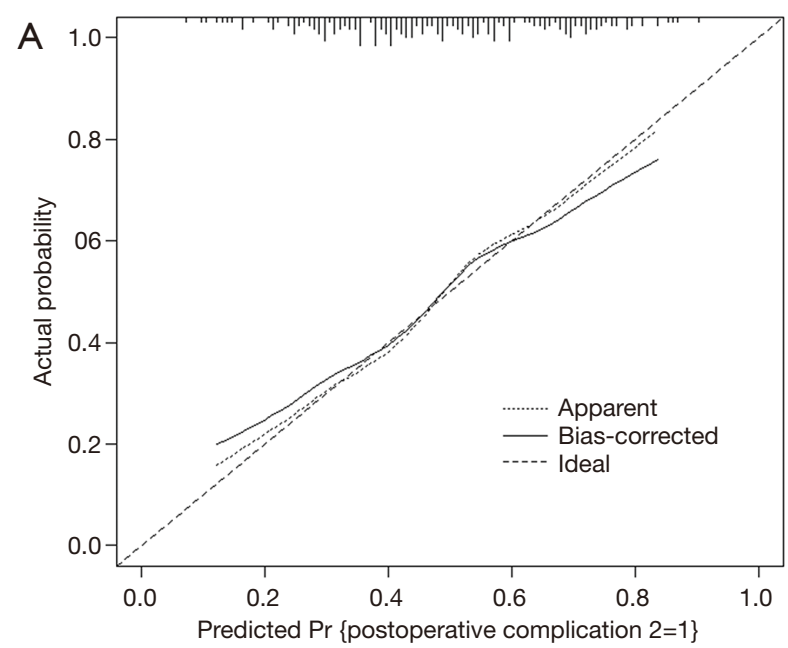

$B=1000$ repetitions, boot Mean absolute error $=0.023 n=223$

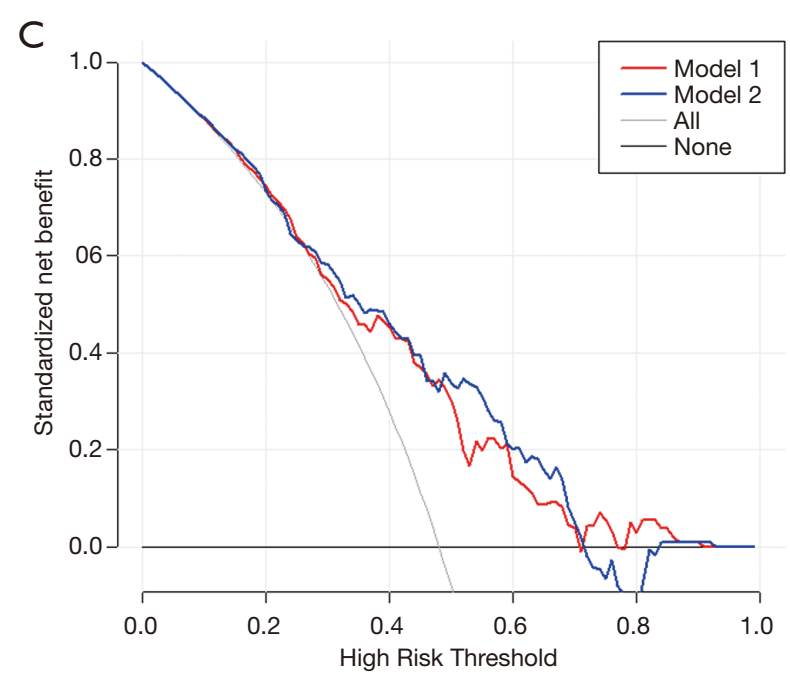

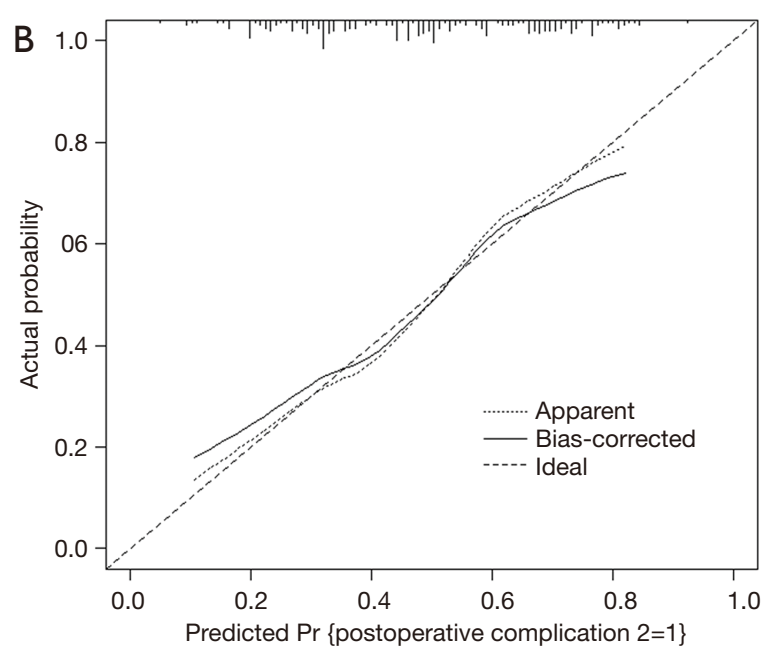

$B=1000$ repetitions, boot Mean absolute error $=0.023 n=223$

Figure 2 Calibration curves of the nomograms without ANS (A) and with ANS (B). Decision curves of the nomograms (C). ANS, albumin/ NLR score.

However, the cut-off values for the inflammatory markers varied among different studies, hampering their clinical applications. In addition, there is limited evidence that compared different inflammatory markers in terms of predictive values for postoperative complications after PD. In this study, ANS exhibited the highest AUC value (0.616) for predicting complications among 15 candidate parameters acquired from routine preoperative examination (Table 2). One rational explanation is that ANS is made up of albumin and NLR, representing the inflammatory response of liver and lymphoid tissue, respectively. Thus, ANS magnifies the predictive value of inflammatory markers for complications. In addition, the AUC value of NLR was higher than PLR in the present study, which was consistent with a previous study showing that NLR was a significant predictor of severe postoperative complications after PD, rather than PLR and PNI (16). This could be interpreted by the fact that neutrophilia has a faster response to changes than thrombocytosis (32). What's more, T-Bil had the third high value $(0.592)$ for predicting complications (Table 2). For those who suffer from periampullary tumor, obstructive jaundice is the most common symptom causing hyperbilirubinemia. Previous studies showed that hyperbilirubinemia could increase overall postoperative complications after PD $(33,34)$, which could explain the results in the present study. 
In this study, the preoperative ANS was found to be independent risk factors for postoperative complications after PD. Subsequently, incorporating ANS into the AICbased multivariable analysis contributed to the increase C-index of 0.725 , compare to 0.710 excluding ANS in the multivariable analysis. What's more, the result of calibration curve as well as NRI indicated that using ANS could significantly improve the predictive ability of the nomogram for postoperative complications. It is wellknown that Albumin exerts an important role in maintaining stable plasma colloid osmotic pressure and combining with many substances as a transport protein (35). Preoperative hypoalbuminemia is usually related to poor nutritional status and is found to be an independent risk factor for increased mortality and morbidity in patients $(36,37)$. On the other hand, previous studies have shown that an elevated NLR is associated with increased morbidity or mortality of patients with different diseases $(16,17,32,38)$. An increased NLR indicates an imbalance between the innate and adaptive immune systems, causing increased proinflammatory cytokine systemically (39). Even though the potential mechanisms between inflammation and complications remain unclear, but these findings in the present study demonstrated that ANS can be employed as an independent predictive factor for complications after PD.

Apart from the ANS, LDH was also incorporated into the nomograms through AIC-based multivariable logistic regression in the present study. The elevation of preoperative $\mathrm{LDH}$ has been demonstrated to be a prognostic factor for the prognosis of patients with pancreatic ductal adenocarcinoma after PD (40). In addition, LDH is a nonspecific inflammatory biomarker, which has been shown to be a powerful predictor for pneumonia with different causes (41-43). In this study, pneumonia is one of the most common complications with the third highest rate $13.0 \%$, which could partially explain the result that LDH is an independent factor for postoperative complications after PD. Further study is pending to study the association between preoperative LDH and postoperative pneumonia after PD.

In addition to ANS, approach method was found to be another significant risk factor for postoperative complication after PD in this study (Table 4). All of the Whipple PDs in this study were performed by the same surgeon who has performed $>100$ PDs over the previous 3 years before 2014 and therefore, was considered to be beyond the learning curve, which was recorded in a multicenter analysis (13). Laparoscopic PD is being increasingly used worldwide. In this study, there was a rising tendency that choosing total laparoscopy as the approach method (Figure S1). Comparisons for the risk of complications between laparoscopic and open PD differs among different studies. While some studies have reported higher rates of complications after laparoscopic PD relative to open PD (44-46), several other studies have shown the opposite results $(47,48)$. In this study, compared to those with laparotomy, patients underwent total laparoscopy had significant lower complication rate $(39.6 \%$ vs. $67.7 \%$; $\mathrm{P}<0.001)$. It could be partially explained by the fact that high-volume center and surgeon experiencing in minimally invasive surgery were reported to associated with lower risk of complication after laparoscopic PD (13). In addition, laparoscopic PD is applied routinely for periampullary lesions in our hospital, apart from some reasons (included large lesions, serious local inflammatory adhesions) leading to difficulty in minimally invasive surgery. This could result in lower incidence of complications after laparoscopic PD.

In order to evaluate the clinical utility of the nomograms in decision-making, the DCA for both nomograms were employed. The DCA showed that both nomograms were useful to predict postoperative complications after PD. More importantly, the nomogram incorporating ANS had higher net benefit compared to the one without ANS. This result indicated that combing inflammatory marker with other risk factors could improve patient outcomes based on nomogram-assisted decision.

There are several limitations in this study. Firstly, nomograms were constructed based on a retrospective study of medical records from a single institution. It is necessary to carry out prospective multicenter study to verify the results of this study. Secondly, the number of patients included in the study is relatively small, which could cause limited statistical power. In future studies, we will increase the sample volume to verify our predictive model.

\section{Conclusions}

In the present study, the preoperative ANS was found to be the most predictive systemic inflammatory marker for postoperative complications after PD. The nomogram with the ANS was built and shown to have better accuracy and superior clinical benefit for predicting postoperative complications, compared to the one without ANS. This nomogram could be applied to the informed consent process before PD and may facilitate physicians' decisionmaking. 


\section{Acknowledgments}

The authors acknowledge the support of Dr. Zhu Liang from The Third Affiliated Hospital of Guangzhou Medical University for assistance in the editing of this manuscript. Funding: This work was supported by the Guangzhou Science and Technology Project [No. 202002020002 to Minghui Cao].

\section{Footnote}

Reporting Checklist: The authors have completed the STROBE reporting checklist. Available at http://dx.doi. org/10.21037/gs-20-789

Data Sharing Statement: Available at http://dx.doi. org/10.21037/gs-20-789

Conflicts of Interest: All authors have completed the ICMJE uniform disclosure form (available at http://dx.doi. org/10.21037/gs-20-789). The authors have no conflicts of interest to declare.

Ethical Statement: The authors are accountable for all aspects of the work in ensuring that questions related to the accuracy or integrity of any part of the work are appropriately investigated and resolved. The study was conducted in accordance with the Declaration of Helsinki (as revised in 2013). The study was approved by institutional ethics committee of Sun Yat-Sen Memorial Hospital (NO. SYSEC-KY-KS-2020-186) and individual consent for this retrospective analysis was waived.

Open Access Statement: This is an Open Access article distributed in accordance with the Creative Commons Attribution-NonCommercial-NoDerivs 4.0 International License (CC BY-NC-ND 4.0), which permits the noncommercial replication and distribution of the article with the strict proviso that no changes or edits are made and the original work is properly cited (including links to both the formal publication through the relevant DOI and the license). See: https://creativecommons.org/licenses/by-nc-nd/4.0/.

\section{References}

1. Karim SAM, Abdulla KS, Abdulkarim QH, et al. The outcomes and complications of pancreaticoduodenectomy (Whipple procedure): Cross sectional study. Int J Surg
2018;52:383-7.

2. Dai J, Jiang Y, Fu D. Reducing postoperative complications and improving clinical outcome: Enhanced recovery after surgery in pancreaticoduodenectomy - A retrospective cohort study. Int J Surg 2017;39:176-81.

3. Yuan F, Essaji Y, Belley-Cote E, et al. Postoperative complications in elderly patients following pancreaticoduodenectomy lead to increased postoperative mortality and costs. A retrospective cohort study. Int J Surg 2018;60:204-9.

4. Aoki S, Miyata H, Konno H, et al. Risk factors of serious postoperative complications after pancreaticoduodenectomy and risk calculators for predicting postoperative complications: a nationwide study of 17,564 patients in Japan. J Hepatobiliary Pancreat Sci 2017;24:243-51.

5. Kosaka H, Kuroda N, Suzumura K, et al. Multivariate logistic regression analysis for prediction of clinically relevant pancreatic fistula in the early phase after pancreaticoduodenectomy. J Hepatobiliary Pancreat Sci 2014;21:128-33.

6. Futagawa Y, Kanehira M, Furukawa K, et al. Impact of delayed gastric emptying after pancreaticoduodenectomy on survival. J Hepatobiliary Pancreat Sci 2017;24:466-74.

7. Brown EG, Yang A, Canter RJ, et al. Outcomes of pancreaticoduodenectomy: where should we focus our efforts on improving outcomes? JAMA Surg 2014;149:694-9.

8. Wang J, Ma R, Churilov L, et al. The cost of perioperative complications following pancreaticoduodenectomy: A systematic review. Pancreatology 2018;18:208-20.

9. Wang J, Ma R, Eleftheriou P, et al. Health economic implications of complications associated with pancreaticoduodenectomy at a University Hospital: a retrospective cohort cost study. HPB (Oxford) 2018;20:423-31.

10. Lu JW, Ding HF, Wu XN, et al. Intraabdominal hemorrhage following 739 consecutive pancreaticoduodenectomy: Risk factors and treatments. J Gastroenterol Hepatol 2019;34:1100-7.

11. Yamamoto Y, Sakamoto Y, Nara S, et al. A Preoperative Predictive Scoring System for Postoperative Pancreatic Fistula after Pancreaticoduodenectomy. World J Surg 2011;35:2747-55.

12. Ellis RJ, Gupta AR, Hewitt DB, et al. Risk factors for postpancreaticoduodenectomy delayed gastric emptying in the absence of pancreatic fistula or intra-abdominal infection. J Surg Oncol 2019;119:925-31. 
13. Wang M, Peng B, Liu J, et al. Practice Patterns and Perioperative Outcomes of Laparoscopic Pancreaticoduodenectomy in China: A Retrospective Multicenter Analysis of 1029 Patients. Ann Surg 2021;273:145-53.

14. Sakamoto T, Yagyu Y, Uchinaka E, et al. Predictive Significance of C-reactive Protein-to-albumin Ratio for Postoperative Pancreatic Fistula After Pancreaticoduodenectomy. Anticancer Res 2019;39:6283-90.

15. Watanabe J, Otani S, Sakamoto T, et al. Prognostic indicators based on inflammatory and nutritional factors after pancreaticoduodenectomy for pancreatic cancer. Surg Today 2016;46:1258-67.

16. Ida M, Tachiiri Y, Sato M, et al. Neutrophil-tolymphocyte ratio as indicator to severe complication after pancreaticoduodenectomy or distal pancreatectomy. Acta Anaesth Scand 2019;63:739-44.

17. Shen CJ, Miao T, Wang Z, et al. Predictive value of postoperative neutrophil/lymphocyte count ratio for surgical site infection in patients following posterior lumbar spinal surgery. Int Immunopharmacol 2019;74:105705.

18. Takeuchi H, Kawanaka H, Fukuyama S, et al. Comparison of the prognostic values of preoperative inflammationbased parameters in patients with breast cancer. Plos One 2017;12:e0177137.

19. Salive ME, Cornoni-Huntley J, Phillips CL, et al. Serum albumin in older persons: relationship with age and health status. J Clin Epidemiol 1992;45:213-21.

20. Ng TP, Feng L, Niti M, et al. Albumin, haemoglobin, $\mathrm{BMI}$ and cognitive performance in older adults. Age Ageing 2008;37:423-9.

21. Adogwa O, Martin J, Huang K, et al. Preoperative serum albumin level as a predictor of postoperative complication after spine fusion. Spine (Phila Pa 1976) 2014;39:1513-9.

22. Liu Z, Wu H, Liufu N, et al. Development and validation of a nomogram incorporating selected systemic inflammation-based prognostic marker for complication prediction after vascularized fibula flap reconstruction. Oral Oncol 2019;99:104467.

23. Sun X, Wang J, Liu J, et al. Albumin concentrations plus neutrophil lymphocyte ratios for predicting overall survival after curative resection for gastric cancer. Onco Targets Ther 2016;9:4661-9.

24. Kao HK, Löfstrand J, Loh CY, et al. Nomogram based on albumin and neutrophil-to-lymphocyte ratio for predicting the prognosis of patients with oral cavity squamous cell carcinoma. Sci Rep 2018;8:13081.

25. Kim Y, Bagante F, Gani F, et al. Nomogram to predict perioperative blood transfusion for hepatopancreaticobiliary and colorectal surgery. Br J Surg 2016;103:1173-83.

26. Zhang H, Feng $\mathrm{Y}$, Zhao J, et al. Total laparoscopic pancreaticoduodenectomy versus open pancreaticoduodenectomy (TJDBPS01): study protocol for a multicentre, randomised controlled clinical trial. BMJ Open 2020;10:e033490.

27. Bassi C, Marchegiani G, Dervenis C, et al. The 2016 update of the International Study Group (ISGPS) definition and grading of postoperative pancreatic fistula: 11 Years After. Surgery 2017;161:584-91.

28. Wente MN, Bassi C, Dervenis C, et al. Delayed gastric emptying (DGE) after pancreatic surgery: A suggested definition by the International Study Group of Pancreatic Surgery (ISGPS). Surgery 2007;142:761-8.

29. Wente MN, Veit JA, Bassi C, et al. Postpancreatectomy hemorrhage (PPH): an International Study Group of Pancreatic Surgery (ISGPS) definition. Surgery 2007;142:20-5.

30. Clavien PA, Barkun J, de Oliveira ML, et al. The ClavienDindo classification of surgical complications: five-year experience. Ann Surg 2009;250:187-96.

31. Mo S, Dai $W$, Xiang $W$, et al. Predictive factors of synchronous colorectal peritoneal metastases: Development of a nomogram and study of its utilities using decision curve analysis. Int J Surg 2018;54:149-55.

32. Bhatti I, Peacock O, Lloyd G, et al. Preoperative hematologic markers as independent predictors of prognosis in resected pancreatic ductal adenocarcinoma: neutrophil-lymphocyte versus platelet-lymphocyte ratio. Am J Surg 2010;200:197-203.

33. De Pastena M, Marchegiani G, Paiella S, et al. Impact of preoperative biliary drainage on Postoperative outcome after pancreaticoduodenectomy: An analysis of 1500 consecutive cases. Dig Endosc 2018;30:777-84.

34. Shen Z, Zhang J, Zhao S, et al. Preoperative biliary drainage of severely obstructive jaundiced patients decreases overall postoperative complications after pancreaticoduodenectomy: A retrospective and propensity score-matched analysis. Pancreatology 2020;20:529-36.

35. Quinlan GJ, Martin GS, Evans TW. Albumin: biochemical properties and therapeutic potential. Hepatology 2005;41:1211-9.

36. Guidet B, Mosqueda GJ, Priol G, et al. The COASST study: cost-effectiveness of albumin in severe sepsis and septic shock. J Crit Care 2007;22:197-203.

37. Daley J, Khuri S, Henderson W, et al. Risk adjustment 
of the postoperative morbidity rate for the comparative assessment of the quality of surgical care: results of the National Veterans Affairs Surgical Risk Study. J Am Coll Surg 1997;185:328-40.

38. Forget P, Dinant V, De Kock M. Is the Neutrophilto-Lymphocyte Ratio more correlated than C-reactive protein with postoperative complications after major abdominal surgery? Peer J 2015;3:e713.

39. Motomura T, Shirabe K, Mano Y, et al. Neutrophillymphocyte ratio reflects hepatocellular carcinoma recurrence after liver transplantation via inflammatory microenvironment. J Hepatol 2013;58:58-64.

40. Ji F, Fu S, Guo Z, et al. Prognostic value of combined preoperative lactate dehydrogenase and alkaline phosphatase levels in patients with resectable pancreatic ductal adenocarcinoma. Medicine (Baltimore) 2016;95:e4065.

41. Ding CY, Peng L, Lin YX, et al. Elevated Lactate Dehydrogenase Level Predicts Postoperative Pneumonia in Patients with Aneurysmal Subarachnoid Hemorrhage. World Neurosurg 2019;129:e821-30.

42. Breuer O, Picard E, Benabu N, et al. Predictors of Prolonged Hospitalizations in Pediatric Complicated Pneumonia. Chest 2018;153:172-80.

Cite this article as: Huang $\mathrm{H}$, Wang C, Ji F, Han Z, Xu H, Cao M. Nomogram based on albumin and neutrophil-tolymphocyte ratio for predicting postoperative complications after pancreaticoduodenectomy. Gland Surg 2021;10(3):877-891. doi: $10.21037 / g s-20-789$
43. Tao RJ, Luo XL, Xu W, et al. Viral infection in community acquired pneumonia patients with fever: a prospective observational study. J Thorac Dis 2018;10:4387-95.

44. Adam MA, Choudhury K, Dinan MA, et al. Minimally Invasive Versus Open Pancreaticoduodenectomy for Cancer: Practice Patterns and Short-term Outcomes Among 7061 Patients. Ann Surg 2015;262:372-7.

45. Sharpe SM, Talamonti MS, Wang CE, et al. Early National Experience with Laparoscopic Pancreaticoduodenectomy for Ductal Adenocarcinoma: A Comparison of Laparoscopic Pancreaticoduodenectomy and Open Pancreaticoduodenectomy from the National Cancer Data Base. J Am Coll Surg 2015;221:175-84.

46. Huang L, Tian $\mathrm{Y}, \mathrm{Wu}$ J, et al. The effectiveness, risks and improvement of laparoscopic pancreaticoduodenectomy during the learning curve: a propensity score-matched analysis. Gland Surg 2020;9:985-99.

47. Yoo D, Song K, Lee J, et al. A Comparative Study of Laparoscopic versus Open Pancreaticoduodenectomy for Ampulla of Vater Carcinoma. J Clin Med 2020;9:2214.

48. Hendi M, Mou Y, Lu C, et al. Laparoscopic pancreaticodoudenectomy: An excellent approach in elderly patients, a multicenter, comparative study. Medicine (Baltimore) 2020;99:e22175. 
Supplementary

Table S1 Complications stratified by approach method

\begin{tabular}{|c|c|c|c|}
\hline & Total laparoscopy $(n=139)$ & Open $(n=65)$ & $\begin{array}{l}\text { Conversion to laparotomy } \\
\qquad(\mathrm{n}=19)\end{array}$ \\
\hline Pancreatic fistula & $18(12.9 \%)$ & $18(27.7 \%)$ & $2(10.5 \%)$ \\
\hline Biochemical fistula & 2 & 2 & 1 \\
\hline Grade B & 14 & 13 & 1 \\
\hline Grade C & 2 & 3 & 0 \\
\hline Delayed gastric emptying & $8(5.8 \%)$ & $10(15.4 \%)$ & $3(15.8 \%)$ \\
\hline Grade A & 1 & 3 & 1 \\
\hline Grade B & 4 & 1 & 1 \\
\hline Grade C & 3 & 6 & 1 \\
\hline Bile leakage & $6(4.3 \%)$ & $6(9.2 \%)$ & $2(10.5 \%)$ \\
\hline Postoperative bleeding & $11(7.9 \%)$ & $7(10.8 \%)$ & $2(10.5 \%)$ \\
\hline Grade B & 5 & 4 & 2 \\
\hline Grade C & 6 & 3 & 0 \\
\hline $\begin{array}{l}\text { Gastrointestinal anastomotic } \\
\text { obstruction }\end{array}$ & $1(0.7 \%)$ & $0(0 \%)$ & $1(5.3 \%)$ \\
\hline Wound infection & $0(0 \%)$ & $1(1.5 \%)$ & $0(0 \%)$ \\
\hline Wound dehiscence & $1(0.7 \%)$ & $0(0 \%)$ & $0(0 \%)$ \\
\hline Abdominal infection & $18(12.9 \%)$ & $16(24.6 \%)$ & $2(10.5 \%)$ \\
\hline Sepsis & $4(2.9 \%)$ & $2(3.1 \%)$ & $1(5.3 \%)$ \\
\hline Pneumonia & $17(12.2 \%)$ & $10(15.4 \%)$ & $2(10.5 \%)$ \\
\hline Urinary tract infection & $1(0.7 \%)$ & $1(1.5 \%)$ & $0(0 \%)$ \\
\hline Acute heart failure & $1(0.7 \%)$ & $1(1.5 \%)$ & $0(0 \%)$ \\
\hline Acute liver failure & $2(1.4 \%)$ & $0(0 \%)$ & $0(0 \%)$ \\
\hline
\end{tabular}

Complication was defined as a Clavien-Dindo classification grade II or higher complication.

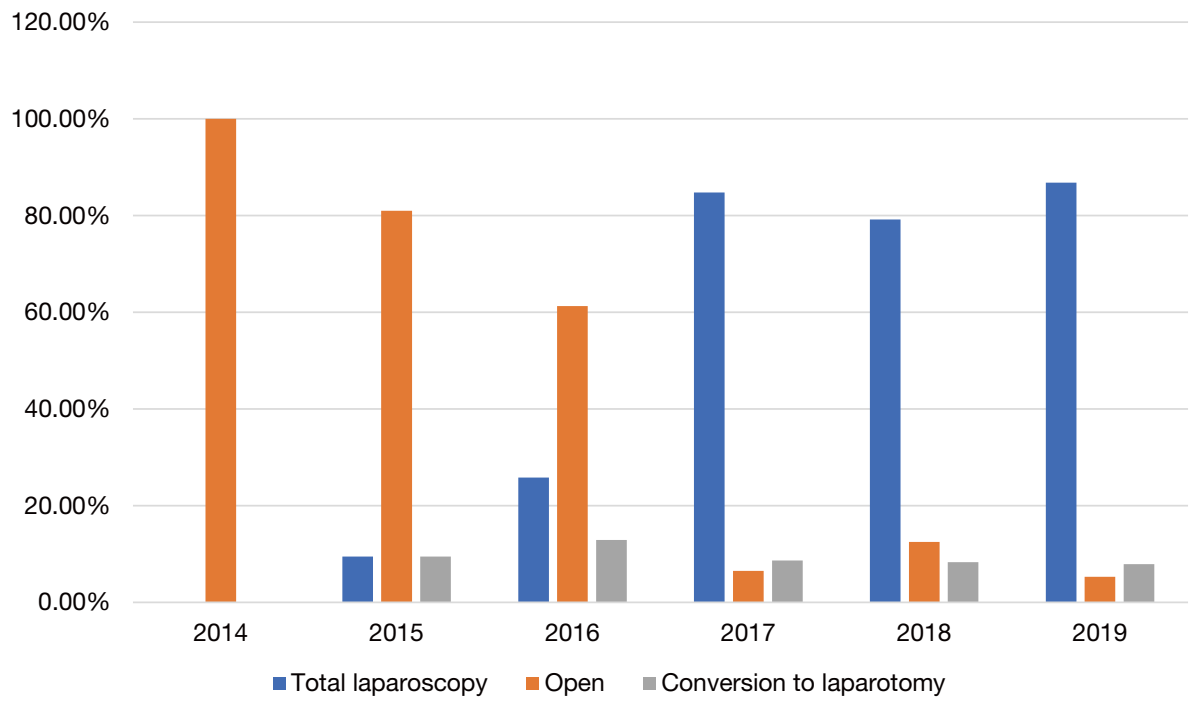

Figure S1 Approach methods over time. 